\title{
Glycogen Storage Disease Type VII
}

National Cancer Institute

\section{Source}

National Cancer Institute. Glycogen Storage Disease Type VII. NCI Thesaurus. Code C118437.

A rare, autosomal recessive inherited metabolic disorder caused by mutation in the PFKM gene. It results in the deficiency of the M subunit of the phosphofructokinase enzyme. It is characterized by the presence of muscle pain and weakness and sometimes rhabdomyolysis with myoglobinuria, following exercise. Affected infants develop muscle weakness. Patients with the hemolytic form of this disorder develop hemolytic anemia without signs or symptoms of muscle pain and weakness. 